\title{
Electromagnetic Radiation from Hot and Dense Hadronic Matter
}

\author{
Pradip Roy, Sourav Sarkar and Jan-e Alam \\ Variable Energy Cyclotron Centre, 1/AF Bidhan Nagar, Calcutta 700064 India \\ Bikash Sinha \\ Variable Energy Cyclotron Centre, 1/AF Bidhan Nagar, Calcutta 700064 India \\ Saha Institute of Nuclear Physics, 1/AF Bidhan Nagar, Calcutta 700064 India
}

\begin{abstract}
The modifications of hadronic masses and decay widths at finite temperature and baryon density are investigated using a phenomenological model of hadronic interactions in the Relativistic Hartree Approximation. We consider an exhaustive set of hadronic reactions and vector meson decays to estimate the photon emission from hot and dense hadronic matter. The reduction in the vector meson masses and decay widths is seen to cause an enhancement in the photon production. It is observed that the effect of $\rho$-decay width on photon spectra is negligible. The effects on dilepton production from pion annihilation are also indicated.
\end{abstract}

PACS: 25.75.+r;12.40.Yx;21.65.+f;13.85.Qk

Keywords: Heavy Ion Collisions, Vector Mesons, Self Energy, Thermal Loops, Bose Enhancement, Photons, Dileptons.

\section{INTRODUCTION}

Numerical simulations of QCD (Quantum Chromodynamics) equation of state on the lattice predict that at very high density and/or temperature hadronic matter undergoes a phase transition to Quark Gluon Plasma (QGP) [1],22. One expects that ultrarelativistic heavy ion collisions might create conditions conducive for the formation and study of QGP. Various model calculations have been performed to look for observable signatures of this state of matter. However, among various signatures of QGP, photons and dileptons are known to be advantageous as these signals participate only in electromagnetic interactions, and therefore, retain their energy and momentum almost undistorted. They thus carry the information of the reaction zone (from where they were produced) rather more effectively unlike the hadrons which are strongly interacting and thus the signals get distorted by final state interaction. The disadvantage with photons is the substantial background from various processes (thermal and non-thermal) [3]. Among these, the contribution from hard QCD processes is well understood in the framework of perturbative QCD and the yield from hadronic decays e. g. $\pi^{0} \rightarrow \gamma \gamma$ can be accounted for by invariant mass analysis. However, photons from the thermalised hadronic gas pose a more difficult task to disentangle. Therefore it is very important to estimate photons from hot and dense hadronic gas along with the possible modification of the hadronic properties.

In ultrarelativistic heavy ion collisions hadronic matter is formed after a phase transition from QGP. Even if such a phase transition does not occur, realisation of hadronic matter at high temperature $(\sim 150-200 \mathrm{MeV})$ and/or baryon density ( a few times normal nuclear matter density) is inevitable. As a result the study of hadronic interactions at high temperature and density assumes great significance. Such a study is also important in order to understand the properties of compact stellar objects such as neutron stars where densities $\sim$ five to ten times normal nuclear matter density are likely to be encountered. There are several other aspects where medium effects may play an important role. For example spontaneously broken chiral symmetry of the normal hadronic world is expected to be restored at high temperature and density [4] and this will be reflected in the thermal shift of the hadron masses. These modifications can be studied by analysing photon, dilepton as well as hadronic spectra.

Progress in our understanding of hot and dense hadronic matter has been retarded since the underlying theory for strong interaction, QCD, is nonperturbative at low energy regime. Because of this severe constraint considerable amount of work has been done on model building (see e.g. Ref. [5]) in order to study the low energy hadronic states. 
Various investigations have addressed the issue of temperature and density dependence of hadronic masses within different models over the past several years. Hatsuda and collaborators [6] and Brown [7] have used the QCD sum rules at finite temperature and density to study the effective masses of the hadrons. Brown and Rho [8] also argued that requiring chiral symmetry (in particular the QCD trace anomaly) yield an approximate scaling relation between various effective hadronic masses, $m_{N}^{*} / m_{N} \sim m_{\sigma}^{*} / m_{\sigma} \sim m_{\rho, \omega}^{*} / m_{\rho, \omega} \sim f_{\pi}^{*} / f_{\pi}$ which implies, that all hadronic masses decrease with temperature. The nonlinear sigma model, claimed to be the closest low energy description of QCD [5] however, shows the opposite trend, i.e. $m_{\rho}^{*}$ increasing with temperature [9]. Thus, there exists a lot of controversy in the literature about the finite temperature properties of hadrons.

The nucleon-nucleon interaction is well described by semi-phenomenological one-boson-exchange models. Inclusion of heavier mesons e. g. rho, omega and other multipion resonant states in the one-pion exchange model gives a good description of low energy $N N$ scattering data. However, in symmetric nuclear matter the interaction mediated by pion and rho exchange averages out to zero. Therefore, many of the observed properties of hadronic interactions at low energy can be understood by considering only omega and sigma meson exchange 10].

The change in the masses and decay widths of vector mesons propagating in a medium occurs due to its interaction with the real and virtual excitations in the medium. In Mean Field Theory (MFT) the condensed scalar field is responsible for the modification of the nucleon mass. In other words the nucleon mass changes due to the contribution from scalar tadpole diagrams in the nucleon self energy 10,11]. The vector meson mass gets shifted due to the decrease of the nucleon mass which appears through thermal loops in the vector meson self energy. The response of the nuclear system to the external probe is characterised by the imaginary part of the vector meson self energy. The interaction of the vector meson with the real particles (on-shell) present in the medium brings in a small change in the mass of the vector meson but the net reduction in the vector meson mass can be attributed to its interaction with the nucleons in the Dirac sea.

In an earlier calculation [12] we have shown the effects of temperature modified masses and widths on the photon spectra. This has relevance to the conditions likely to be achieved at RHIC and LHC energies. However, due to partial stopping of baryonic matter at the SPS energies, the central region of heavy ion collision can have non-zero baryon density [13]. Hence it is necessary to include finite baryon density effects in the hadronic properties as well.

In the present calculation we have studied the medium modification of hadron properties both at finite temperature and baryon density. In addition to this we have also included the effects of vacuum fluctuations in the nucleon self energy. The photon emission rates are then estimated with medium modified mass and decay width of hadrons. Possible effects on the dilepton emission rate have also been illustrated.

We organise the paper as follows. In Section II we calculate the temperature and density dependent properties of vector mesons within the framework of Quantum Hadrodynamics (QHD). In Section III we discuss photon production rates from hot and dense matter. Section IV is devoted to discuss the results of our calculations. In Section V we present a summary and discussions.

\section{MEDIUM EFFECTS}

At non-zero temperature and density the pole of the propagator gets shifted due to interactions with real and virtual excitations present in the system. Such a modification can be studied through the Dyson - Schwinger equation. 


\section{IIa. Nucleons}

In the Relativistic Hartree Approximation (RHA) the full nucleon propagator is given by,

$$
G^{H}(k)=G^{0}(k)+G^{0}(k) \Sigma^{H}(k) G^{H}(k)
$$

where $\Sigma^{H}(k)$ is the nucleon self energy which contains contributions from both scalar $\left(\Sigma_{s}\right)$ and vector $\left(\Sigma_{v}^{\mu}\right)$ tadpole diagrams [10,11] and is given by

$$
\Sigma^{H}=\Sigma_{s}^{H}-\gamma^{\mu} \Sigma_{\mu v}^{H}
$$

where,

$$
\Sigma_{s}^{H}=i \frac{g_{s}^{2}}{m_{s}^{2}} \int \frac{d^{4} q}{(2 \pi)^{4}} \operatorname{Tr}\left[G^{H}(q)\right]+\Sigma_{s}^{\mathrm{CTC}}
$$

and,

$$
\Sigma_{\mu v}^{H}=i \frac{g_{v}^{2}}{m_{v}^{2}} \int \frac{d^{4} q}{(2 \pi)^{4}} \operatorname{Tr}\left[\gamma_{\mu} G^{H}(q)\right]
$$

Here, $m_{s}\left(m_{v}\right)$ is the mass of the neutral scalar (vector) meson, and, the nucleon interacts via the exchange of scalar (vector) meson with coupling constant $g_{s}\left(g_{v}\right) . \Sigma_{s}^{\mathrm{CTC}}$ is the counter term contribution required to subtract the divergences in the scalar self energy. Since the vector self energy is finite, such a counter term is not required [10,11]. The pole structure of the full nucleon propagator in RHA resembles that of the non-interacting propagator with shifted mass and four-momentum. The full nucleon propagator consists of a medium and a vacuum part. Mean Field Theory is reproduced if one considers only the medium contribution. However, the inclusion of the vacuum part results in divergences. One then introduces counterterms $\left(\Sigma_{s}^{\mathrm{CTC}}\right)$ in order to subtract out the divergences. These constitute the vacuum fluctuation corrections to MFT. Consequently, the effective nucleon mass reads,

$$
\begin{aligned}
\Sigma_{s}^{H} & =M^{*}-M \\
& =-\frac{g_{s}^{2}}{m_{s}^{2}} \frac{4}{(2 \pi)^{3}} \int d^{3} \mathbf{k} \frac{M^{*}}{E^{*}}\left[n_{B}\left(\mu^{*}, T\right)+\bar{n}_{B}\left(\mu^{*}, T\right)\right] \\
& +\frac{g_{s}^{2}}{m_{s}^{2}} \frac{1}{\pi^{2}}\left[M^{* 3} \ln \left(\frac{M^{*}}{M}\right)-M^{2}\left(M^{*}-M\right)\right. \\
& \left.-\frac{5}{2} M\left(M^{*}-M\right)^{2}-\frac{11}{6}\left(M^{*}-M\right)^{3}\right]
\end{aligned}
$$

where,

$$
\begin{aligned}
n_{B}\left(\mu^{*}, T\right) & =\frac{1}{\exp \left[\left(E^{*}-\mu^{*}\right) / T\right]+1} \\
\bar{n}_{B}\left(\mu^{*}, T\right) & =\frac{1}{\exp \left[\left(E^{*}+\mu^{*}\right) / T\right]+1} \\
E^{*} & =\sqrt{\left(\mathbf{k}^{2}+M^{* 2}\right)} \\
\mu^{*} & =\mu-\left(\frac{g_{v}^{2}}{m_{v}^{2}}\right) \rho
\end{aligned}
$$

Here, $\rho$ is the baryon density of the medium and is given by

$$
\rho=\frac{4}{(2 \pi)^{3}} \int d^{3} \mathbf{k}\left[n_{B}\left(\mu^{*}, T\right)-\bar{n}_{B}\left(\mu^{*}, T\right)\right]
$$




\section{IIb. Vector Mesons}

The effective propagator for the vector boson is given by,

$$
D_{\mu \nu}=-\frac{A_{\mu \nu}}{k^{2}-m_{V}^{2}+\Pi_{T}}-\frac{B_{\mu \nu}}{k^{2}-m_{V}^{2}+\Pi_{L}}+\frac{k_{\mu} k_{\nu}}{k^{2} m_{V}^{2}},
$$

where $A_{\mu \nu}$ and $B_{\mu \nu}$ are the projection operators $12, m_{V}$ is the free mass of the vector meson and

$$
\Pi_{T(L)}=\Pi_{T(L)}^{D}+\Pi^{F}
$$

The transverse (longitudinal) component of the self energy, $\Pi_{T(L)}^{D}$ contains both finite temperature and density effects and $\Pi^{F}$ represents the contribution from the Dirac sea with modified nucleon mass. We use the following interaction Lagrangian to evaluate the rho and omega self energies:

$$
\mathcal{L}_{V N N}=g_{V N N}\left(\bar{N} \gamma_{\mu} \tau^{a} N V_{a}^{\mu}-\frac{\kappa_{V}}{2 M} \bar{N} \sigma_{\mu \nu} \tau^{a} N \partial^{\nu} V_{a}^{\mu}\right)
$$

where $V_{a}^{\mu}=\left\{\omega^{\mu}, \vec{\rho}^{\mu}\right\}, M$ is the free nucleon mass, $N$ is the nucleon field and $\tau_{a}=\{1, \vec{\tau}\}$.

The real part of the self energy is responsible for mass shifting and the imaginary part gives the decay width of the vector meson in the medium. The physical mass of the vector meson can be obtained from the pole position of the propagator in the limit $|\mathbf{k}| \rightarrow \mathbf{0} i$. e. from the solution of the longitudinal or transverse dispersion relation in the rest frame of the vector meson as given in appendix A. The rho decay width is calculated from the $\rho \pi \pi$ loop by using Landau - Cutkosky cutting rules at finite temperature, the details being presented in appendix A.

\section{IIc. Spectral Function for Vector Mesons}

The density of a stable hadron of mass $m$ in a thermal bath is completely determined by the temperature, chemical potential and the statistics obeyed by the species through the following equation

$$
\frac{d N}{d^{3} x d^{3} k d s}=\frac{g}{(2 \pi)^{3}} \frac{1}{\exp \left(k_{0}-\mu\right) / T \pm 1} \delta\left(s-m^{2}\right)
$$

where $g$ is the statistical degeneracy and $k_{0}=\sqrt{\vec{k}^{2}+s}$ is the energy of the particle in the rest frame of the thermal bath. The question one would like to ask is - how will eq. (10) be modified if the particle decays within the thermal bath? This is a relevant question for $\rho$ and $\omega$ mesons as their life times $(1.3 \mathrm{fm} / \mathrm{c}$ and $23.5 \mathrm{fm} / \mathrm{c}$ respectively in vacuum) are comparable to the strong interaction time scale and therefore they can decay within the thermal system formed after ultra-relativistic heavy ion collisions. This problem has been addressed by Weldon 14 through the generalisation of Breit-Wigner formula at finite temperature and density and its consequences on the dileptonic decay of vector mesons have been studied in [15. The distribution of an unstable particle in a thermal bath is given by 14

$$
\frac{d N}{d^{3} x d^{3} k d s}=\frac{g}{(2 \pi)^{3}} \frac{1}{\exp \left(k_{0}-\mu\right) / T \pm 1} P(s)
$$

where $P(s)$ is called the spectral function [16 18 can be calculated from the effective thermal propagator. It is given by

$$
P(s)=\left[\frac{1}{\pi} \frac{\operatorname{Im} \Pi}{\left(s-m_{V}^{2}+\operatorname{Re} \Pi\right)^{2}+(\operatorname{Im} \Pi)^{2}}\right]
$$


Equation (11) indicates that to obtain realistic results for the photon production through a reaction involving unstable particle in the external line the finite width of the particle should be taken into account by introducing the spectral representation of the corresponding particle and integrating over $s$ [19]. This is done in our calculation for the unstable vector mesons appearing in the external line in reactions for photon production. In case the unstable particle appears in the internal line the finite width of the particle is taken into account through effective propagators. It is interesting to note that the spectral function reduces to a Dirac delta function $\delta\left(s-m_{V}^{2}+\operatorname{Re} \Pi\right)$ in the limit $\operatorname{Im} \Pi \rightarrow 0$ i.e. when the particle is stable. In the calculation of the imaginary part of the self energy $\operatorname{Im} \Pi$ of $\rho$ say, one must in principle, include all the processes which can create or annihilate a $\rho$ in the thermal bath. However, within the ambit of the model adopted in the present work we have seen that the most dominant contribution to Im $\Pi$ comes from the $\rho-\pi-\pi$ interaction in the temperature and density range of our interest.

\section{PHOTON EMISSION}

To evaluate the photon emission rate from a hadronic gas we model the system as consisting of $\pi, \rho, \omega$ and $\eta$. The relevant vertices for the reactions $\pi \pi \rightarrow \rho \gamma$ and $\pi \rho \rightarrow \pi \gamma$ and the decay $\rho \rightarrow \pi \pi \gamma$ are obtained from the following Lagrangian:

$$
\mathcal{L}=-g_{\rho \pi \pi} \vec{\rho}^{\mu} \cdot\left(\vec{\pi} \times \partial_{\mu} \vec{\pi}\right)-e J^{\mu} A_{\mu}+\frac{e}{2} F^{\mu \nu}\left(\vec{\rho}_{\mu} \times \vec{\rho}_{\nu}\right)_{3},
$$

where $F_{\mu \nu}=\partial_{\mu} A_{\nu}-\partial_{\nu} A_{\mu}$, is the Maxwell field tensor and $J^{\mu}$ is the hadronic part of the electromagnetic current given by

$$
J^{\mu}=\left(\vec{\rho}_{\nu} \times \vec{B}^{\nu \mu}\right)_{3}+\left(\vec{\pi} \times\left(\partial^{\mu} \vec{\pi}+g_{\rho \pi \pi} \vec{\pi} \times \vec{\rho}^{\mu}\right)\right)_{3}
$$

with $\vec{B}_{\mu \nu}=\partial_{\mu} \vec{\rho}_{\nu}-\partial_{\nu} \vec{\rho}_{\mu}-g_{\rho \pi \pi}\left(\vec{\rho}_{\mu} \times \vec{\rho}_{\nu}\right)$. The invariant amplitudes for all these reactions have been listed in the appendix of Ref. [12].

For the sake of completeness we have also considered the photon production due to the reactions $\pi \eta \rightarrow \pi \gamma$, $\pi \pi \rightarrow \eta \gamma$ and the decay $\omega \rightarrow \pi \gamma$ using the following interaction:

$$
\mathcal{L}=\frac{g_{\rho \rho \eta}}{m_{\eta}} \epsilon_{\mu \nu \alpha \beta} \partial^{\mu} \rho^{\nu} \partial^{\alpha} \rho^{\beta} \eta+\frac{g_{\omega \rho \pi}}{m_{\pi}} \epsilon_{\mu \nu \alpha \beta} \partial^{\mu} \omega^{\nu} \partial^{\alpha} \rho^{\beta} \pi+\frac{e m_{\rho}^{2}}{g_{\rho \pi \pi}} A_{\mu} \rho^{\mu}
$$

The last term in the above Lagrangian is written down on the basis of Vector Meson Dominance (VMD) [20]. The invariant amplitudes for the reactions involving the $\eta$ meson are given in appendix B.

The emission rate of a photon of energy $E$ and momentum $\mathbf{p}$ from a thermal system at a temperature $T$ is given by

$$
\begin{aligned}
E \frac{d R}{d^{3} \mathbf{p}}= & \frac{\mathcal{N}}{16(2 \pi)^{7} E} \int_{\left(m_{1}+m_{2}\right)^{2}}^{\infty} d s \int_{t_{\min }}^{t_{\max }} d t|\mathcal{M}|^{2} \int d E_{1} \\
& \times \int d E_{2} \frac{f\left(E_{1}\right) f\left(E_{2}\right)\left[1+f\left(E_{3}\right)\right]}{\sqrt{a E_{2}^{2}+2 b E_{2}+c}}
\end{aligned}
$$

where $\mathcal{M}$ is the invariant amplitude for photon production and

$$
\begin{aligned}
a= & -\left(s+t-m_{2}^{2}-m_{3}^{2}\right)^{2} \\
b= & E_{1}\left(s+t-m_{2}^{2}-m_{3}^{2}\right)\left(m_{2}^{2}-t\right)+E\left[\left(s+t-m_{2}^{2}-m_{3}^{2}\right)\left(s-m_{1}^{2}-m_{2}^{2}\right)\right. \\
& \left.-2 m_{1}^{2}\left(m_{2}^{2}-t\right)\right]
\end{aligned}
$$




$$
\begin{aligned}
c= & -E_{1}^{2}\left(m_{2}^{2}-t\right)^{2}-2 E_{1} E\left[2 m_{2}^{2}\left(s+t-m_{2}^{2}-m_{3}^{2}\right)-\left(m_{2}^{2}-t\right)\left(s-m_{1}^{2}-m_{2}^{2}\right)\right] \\
& -E^{2}\left[\left(s-m_{1}^{2}-m_{2}^{2}\right)^{2}-4 m_{1}^{2} m_{2}^{2}\right]-\left(s+t-m_{2}^{2}-m_{3}^{2}\right)\left(m_{2}^{2}-t\right) \\
& \times\left(s-m_{1}^{2}-m_{2}^{2}\right)+m_{2}^{2}\left(s+t-m_{2}^{2}-m_{3}^{2}\right)^{2}+m_{1}^{2}\left(m_{2}^{2}-t\right)^{2} \\
E_{1 \text { min }}= & \frac{\left(s+t-m_{2}^{2}-m_{3}^{2}\right)}{4 E}+\frac{E m_{1}^{2}}{s+t-m_{2}^{2}-m_{3}^{2}} \\
E_{2 \min }= & \frac{E m_{2}^{2}}{m_{2}^{2}-t}+\frac{m_{2}^{2}-t}{4 E} \\
E_{2 \max }= & -\frac{b}{a}+\frac{\sqrt{b^{2}-a c}}{a} .
\end{aligned}
$$

\section{RESULTS}

\section{IVa. Hadronic Properties in the Medium}

The effective nucleon mass (which appears in the nucleon loop contribution to self energies of the rho and omega mesons) has been calculated, within the framework of the model defined above, in the RHA. The following values of the coupling constants and masses [21] have been used in our calculations: $\kappa_{\rho}=6.1, g_{\rho N N}^{2}=6.91, m_{s}=458 \mathrm{MeV}$, $m_{\rho}=770 \mathrm{MeV}, M=939 \mathrm{MeV}, g_{s}^{2}=54.3, \kappa_{\omega}=0$, and $g_{v}^{2} \equiv g_{\omega N N}^{2}=102$. In Fig. (1) we depict the variation of nucleon mass with temperature for a set of densities. We observe that the nucleon mass falls steadily with density for a fixed temperature. However, the variation with temperature for given values of baryon densities shows interesting features. At zero baryon density the nucleon mass decreases monotonically as a function of temperature, but for finite densities it increases slightly before falling. This trend is similar to that obtained by Li et al [22], and may be attributed to the modification of the Fermi-sea at finite temperature and density. Our calculation shows a 35\% reduction of the effective nucleon mass at $T=160 \mathrm{MeV}$ and two times normal nuclear matter density compared to its free mass. In order to highlight the effect of vacuum fluctuation corrections we compare the MFT results with those obtained using RHA. This is plotted in Fig. (2). We observe that the effect of vacuum fluctuation is substantial for higher values of the baryon density. The contribution of the antinucleons from the Dirac sea is responsible for such an effect.

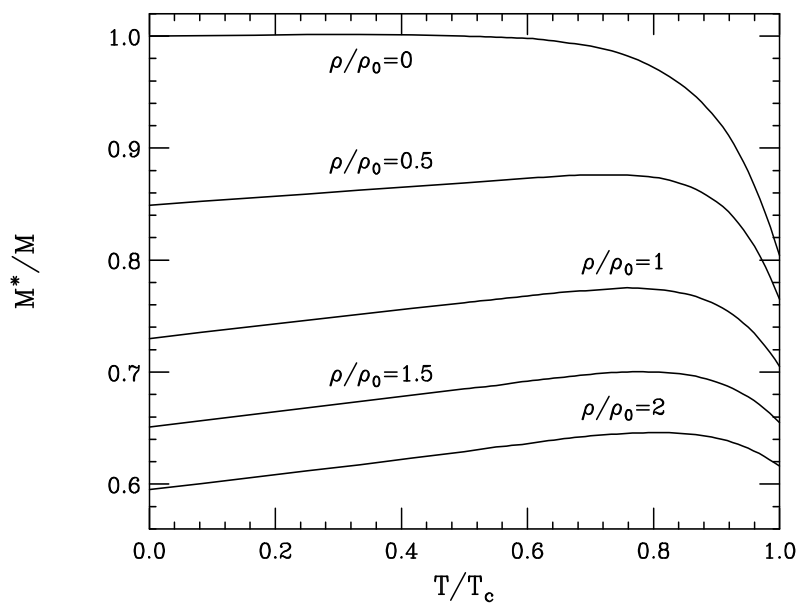

FIG. 1. Variation of nucleon mass with temperature for different values of baryon densities. Here, $\rho_{0}=0.1484 \mathrm{fm}^{-3}$ and $T_{c}=200 \mathrm{MeV}$. 
We now focus our attention to the properties of vector mesons due to $N-N$ interactions at finite temperature and baryon density. The major contribution to the medium effects on the rho and omega mesons, in this approach, arises from the nucleon-loop diagram. For the dressing of internal lines in matter we restrict ourselves to Mean Field Theory (MFT) to avoid a plethora of diagrams and to maintain internal consistency. Since the Walecka model does not have chiral symmetry, it is rather difficult to predict anything reliable on the pion mass in this model, especially in the MFT approximation [10,23]. On the other hand, in the models with chiral symmetry e.g. the Nambu-Jona-Lasinio model, and the linear sigma model with nucleon, it is well-known that the pion mass is almost unchanged in so far as one is in the Nambu-Goldstone phase. This is simply a consequence of the Nambu-Goldstone theorem in medium [24]; we thus adopt the approach of keeping the pion mass constant. The longitudinal and transverse dispersion relations are shown in Fig. (3) for two sets of coupling constants $\left(g_{V}, \kappa_{V}\right)=(10.2,0)$ and $(2.63,6)$ relevant for omega and rho mesons respectively. One observes a small difference between the two modes in case of the omega meson. The vanishing of this difference for the rho meson can be attributed to the smaller vector and larger tensor coupling constants as compared to the omega meson. In Fig. (雨) the effective mass of the rho meson is plotted against temperature for various values of baryon density. We observe that the variation of the rho mass follows qualitatively the same trend as that of the nucleon. In this case, the rho mass decreases by $45 \%$ at $T=160 \mathrm{MeV}$ and two times normal nuclear matter density compared to its free space value. This is due to the fact that the large decrease of the modified Dirac sea contribution to the rho self energy dominates over the in-medium contribution which is seen to increase with temperature. It has been shown 12,25] that the change in the rho mass due to rho pion interaction is negligibly small at non-zero temperature and zero baryon density. In a different model calculation it has been shown by Klingl et al [26] that up to to leading order in density the shift in the rho mass is very small. Therefore the change in rho meson properties due to $\rho-\pi-\pi$ interaction is neglected here. In another approach the in-medium modification in the spectral function of the rho meson was studied [27 29] by including the medium effects on the $\rho-\pi-\pi$ vertex and the pion propagator in the delta-hole model at non-zero density. However, it is observed in these studies that the rho mass remains almost unchanged due to rho-pion interaction up to normal nuclear matter density. Since in this work we restrict our calculations within the realm of MFT, i.e the internal nucleon loop in the rho and omega self energy are modified due to tadpole diagram only, the inclusion of vertex corrections and modification of the pion propagator due to delta-hole excitation will take us beyond MFT and hence are not considered here. However, if one includes the delta-hole polarisation (and $\mathrm{N}-\mathrm{N}$ polarisation also) effects on pions, the rho mass may change from the values obtained here due to $\rho-\pi$ interaction for $\rho>\rho_{0}$. We do not include the delta baryon in the present work.

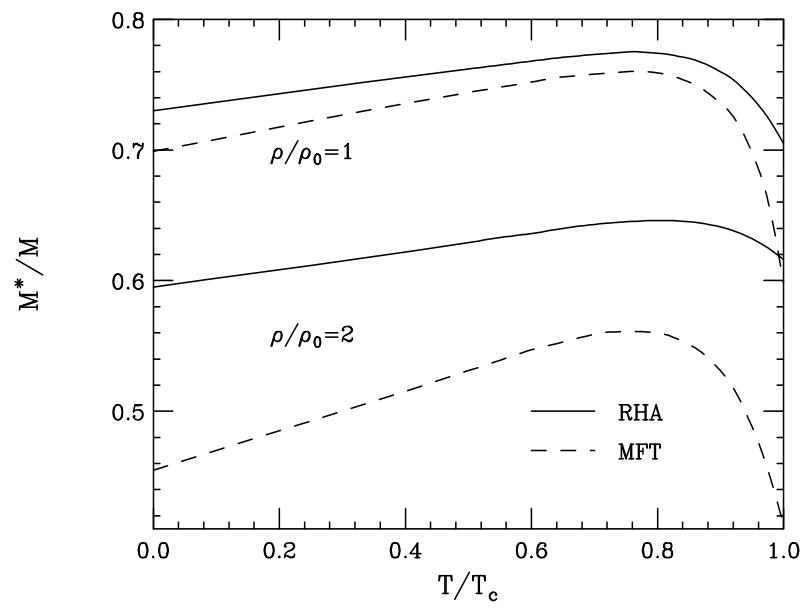

FIG. 2. Same as Fig. 11 with (solid) and without( dashed) vacuum fluctuation corrections to MFT. 


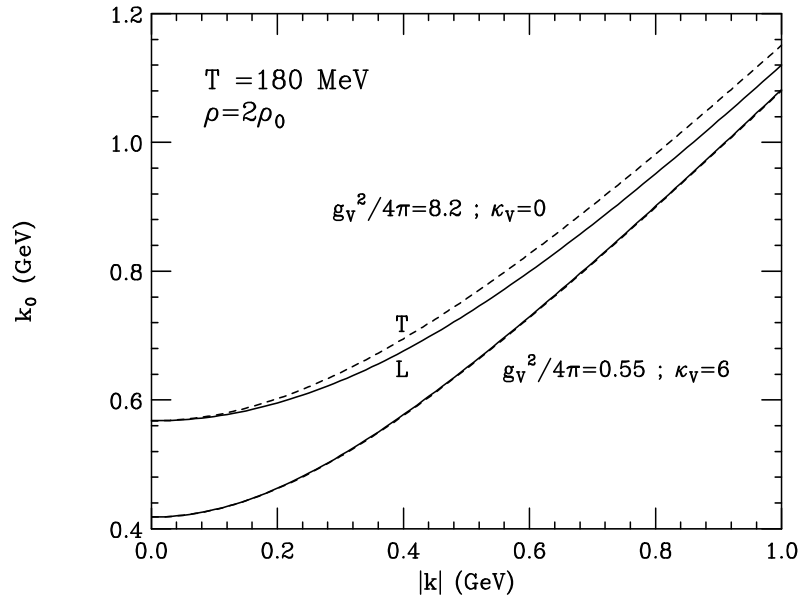

FIG. 3. Longitudinal and transverse dispersion relation for vector mesons.

We evaluate the effective omega mass with the values of the coupling constants mentioned above. We observe in Fig. (5) that the mass of the omega meson as a function of temperature follows qualitatively similar trend as that of the rho meson. The quantitative difference in the rho and omega meson masses is due to the different numerical values of the coupling constants e.g. the tensor interaction is absent in case of the omega meson.

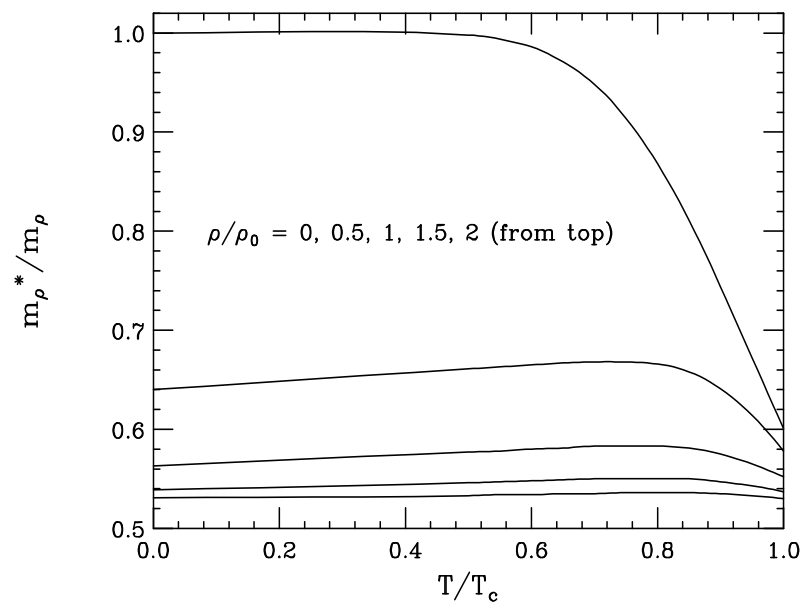

FIG. 4. Variation of rho mass with temperature for various baryon densities.

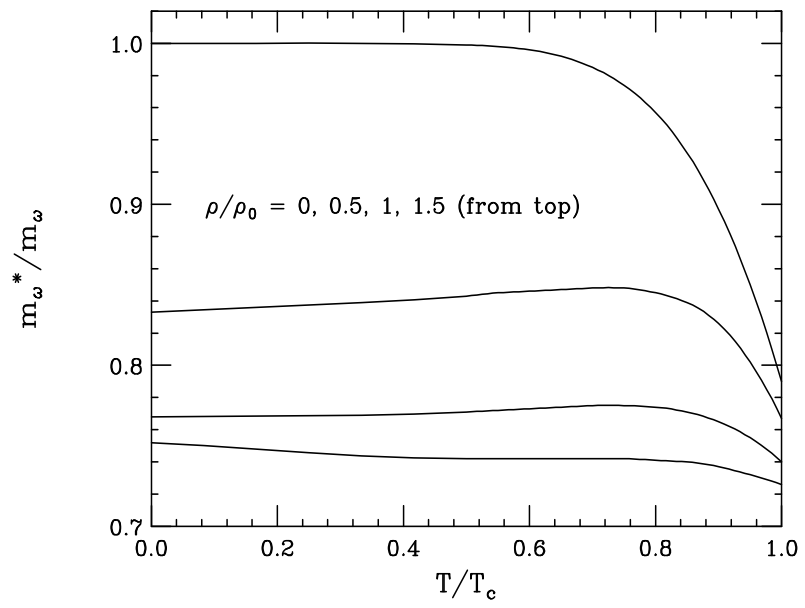

FIG. 5. Variation of omega mass with temperature for various baryon densities. 
Now we demonstrate the in-medium effect on the decay width of rho meson. This is shown in Fig. (6). In this case the observed enhancement of the decay width with temperature at non-zero values of the baryon density is solely due to stimulated emission of pions in the medium. This is just a manifestation of the well known Bose enhancement (BE) effect [12, 30] which is more clearly observed in Fig. (7). We will show in the next section that the BE effect plays an important role in the dilepton spectra.

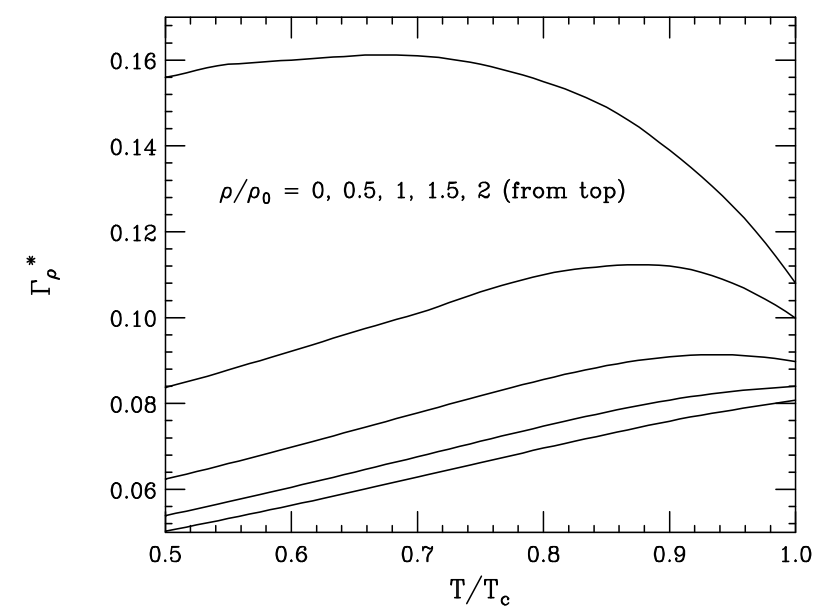

FIG. 6. The $\rho \rightarrow \pi \pi$ decay width as a function of temperature for different values of baryon densities.

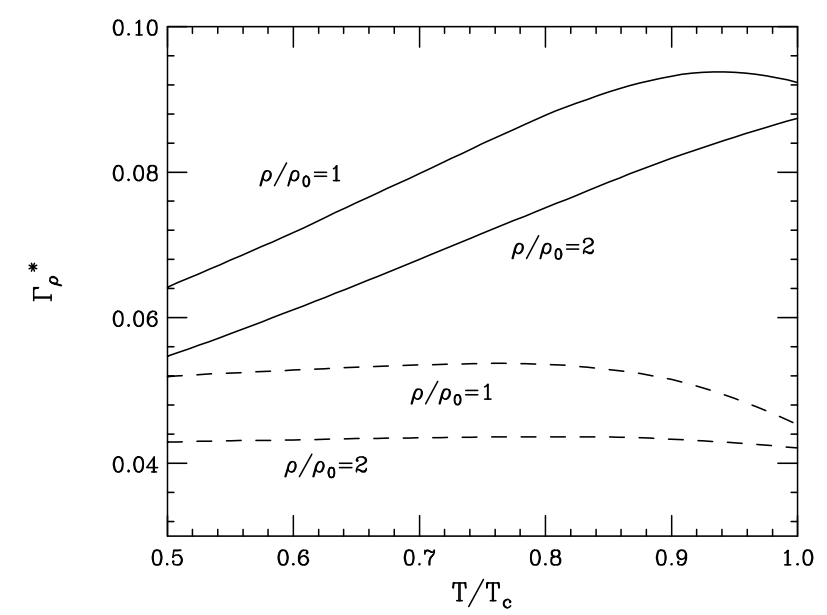

FIG. 7. Same as Fig. 1 with (solid) and without (dashed) BE effect.

\section{IVb. Photons from Hadronic Matter}

In the present section we evaluate the photon spectra from a hot and dense hadronic medium. The medium effects enter through the polarisation functions in the vector meson propagator described by eq.(7). It is well known [31] that the reactions $\pi \rho \rightarrow \pi \gamma, \pi \pi \rightarrow \rho \gamma, \pi \pi \rightarrow \eta \gamma, \pi \eta \rightarrow \pi \gamma$, and the decays $\rho \rightarrow \pi \pi \gamma$ and $\omega \rightarrow \pi \gamma$ are the most important channels for photon production from hadronic matter in the energy regime of our interest. 


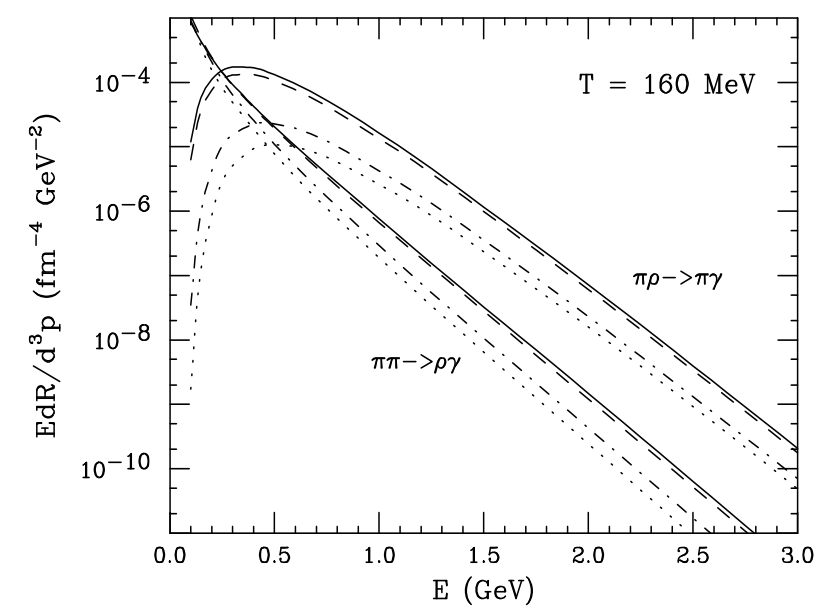

FIG. 8. Photon emission rates as a function of energy of the emitted photon at $T=160 \mathrm{MeV}$. Solid and dashed lines show the results for $\rho / \rho_{0}=2$ and 1 respectively. The dotdashed line represents only finite temperature effects and dotted line shows the result when no medium effect is considered.

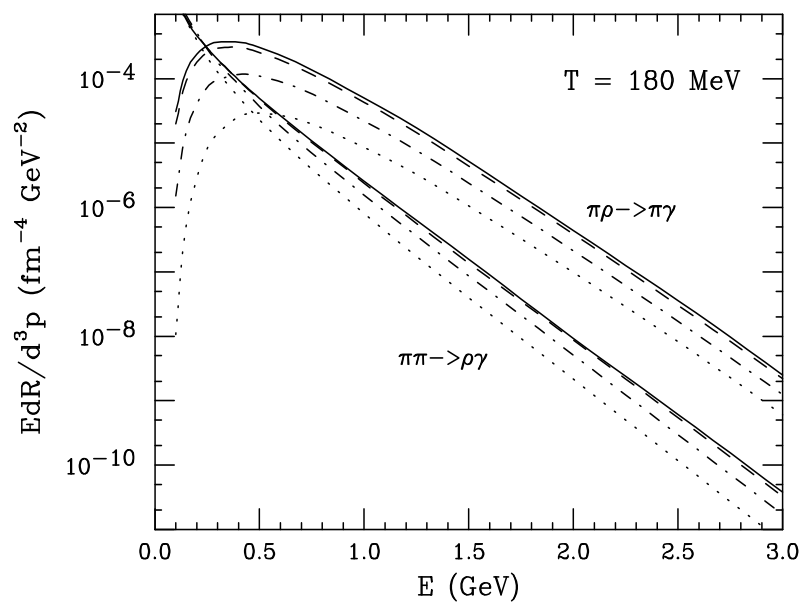

FIG. 9. Same as Fig. 8 at $T=180 \mathrm{MeV}$.

We start with the reactions $\pi \rho \rightarrow \pi \gamma$ and $\pi \pi \rightarrow \rho \gamma$. Fig. (8) shows the photon yields from these reactions as a function of energy at $T=160 \mathrm{MeV}$ for various values of the baryon density. For the channel $\pi \pi \rightarrow \rho \gamma$, we observe that at two times normal nuclear density the emission rate of photons in the energy range 1-3 GeV increases by a factor of $\sim 6$ compared to its value when no medium effect is taken into account. We can understand this enhancement from a simple kinematical argument. For a given center of mass energy of the $\pi-\pi$ system, the energy of a photon can be written as $E_{\gamma}=\left(\sqrt{s}-m_{\rho}^{2} / \sqrt{s}\right) / 2$. One can immediately see from here that the decrease in rho mass enhances the possibility of getting a photon of higher energy. Let us now turn to the reaction $\pi \rho \rightarrow \pi \gamma$. In this case also we observe a similar enhancement in the photon yield at higher energies. However, at lower photon energies $(<1 \mathrm{GeV})$ the enhancement is by a few orders of magnitude. The rho meson in the incident channel in this case appears as a massless boson $(\gamma)$ in the exit channel making available the rest mass energy of the rho to the kinetic energy of the emitted photon. In Fig. (9), the same is shown at a temperature $T=180 \mathrm{MeV}$. The results are qualitatively similar to the previous case with an increase in the absolute value of the photon yield. 


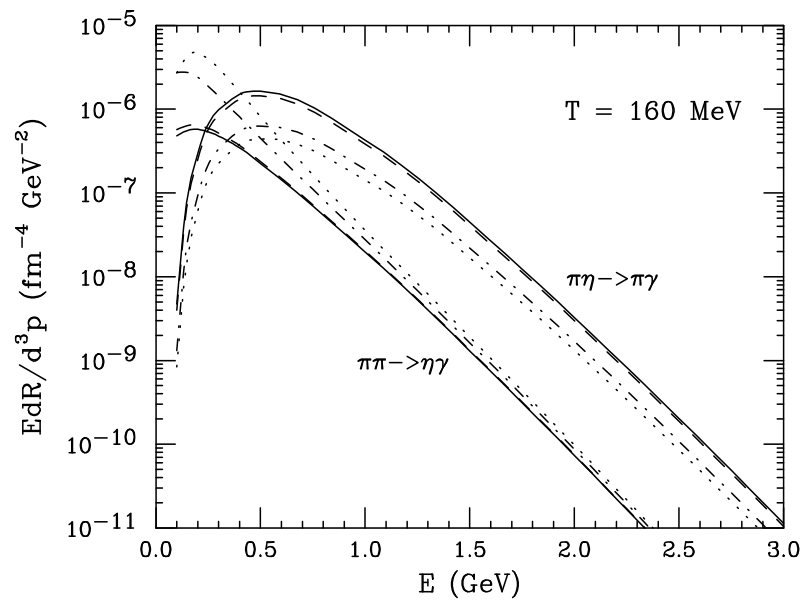

FIG. 10. Same as Fig. $\mathrm{B}$ for the reactions $\pi \eta \rightarrow \pi \gamma$ and $\pi \pi \rightarrow \eta \gamma$.

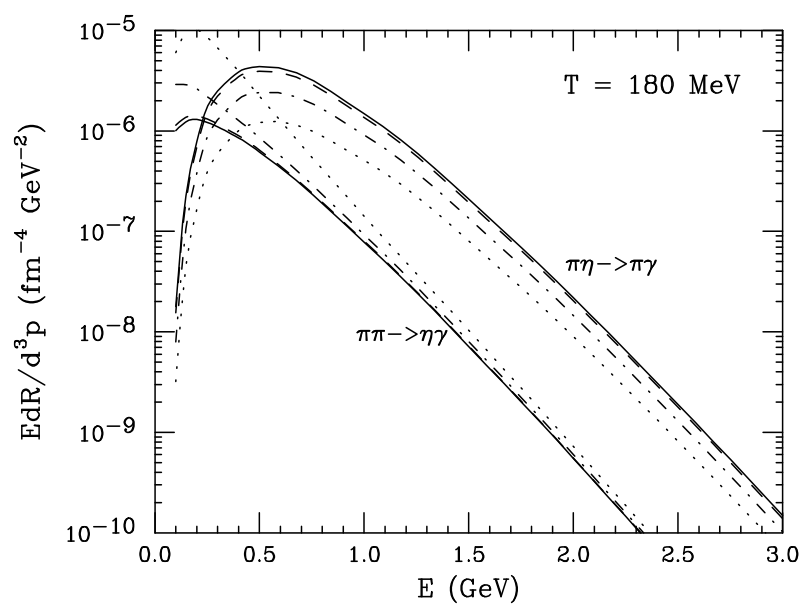

FIG. 11. Same as Fig. 10 at $T=180 \mathrm{MeV}$.

We next consider the reactions $\pi \pi \rightarrow \eta \gamma$, and $\pi \eta \rightarrow \pi \gamma$. In Fig. (10) we plot the photon emission rates from these reactions at $T=160 \mathrm{MeV}$ for different baryon densities. It is interesting to note that with increasing values of the baryon density the yield from the first reaction decreases whereas the opposite occurs for the second reaction. Such a behaviour naturally follows from the rho mass (effective) dependence of the invariant amplitudes (see appendix B). The same features are also observed at $T=180 \mathrm{MeV}$ as shown in Fig. (11). 


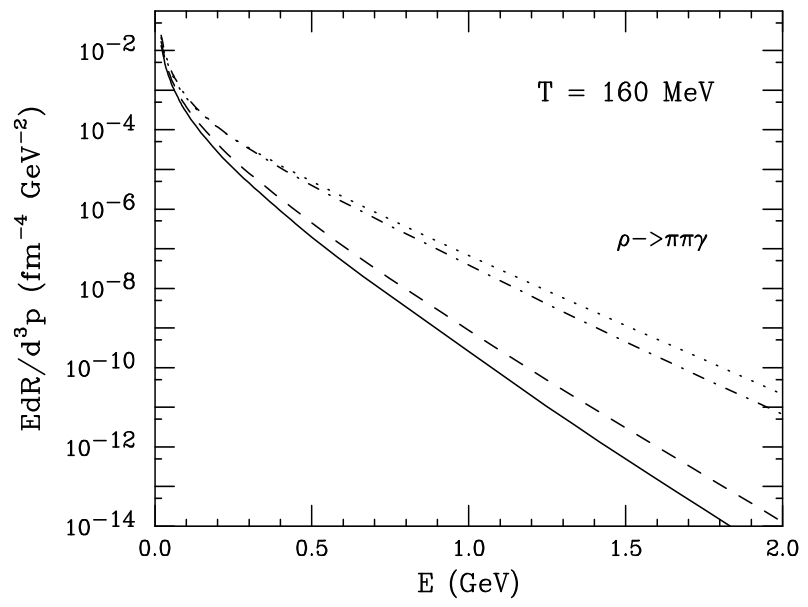

FIG. 12. Same as Fig. $\mathrm{B}$ for the decay $\rho \rightarrow \pi \pi \gamma$.

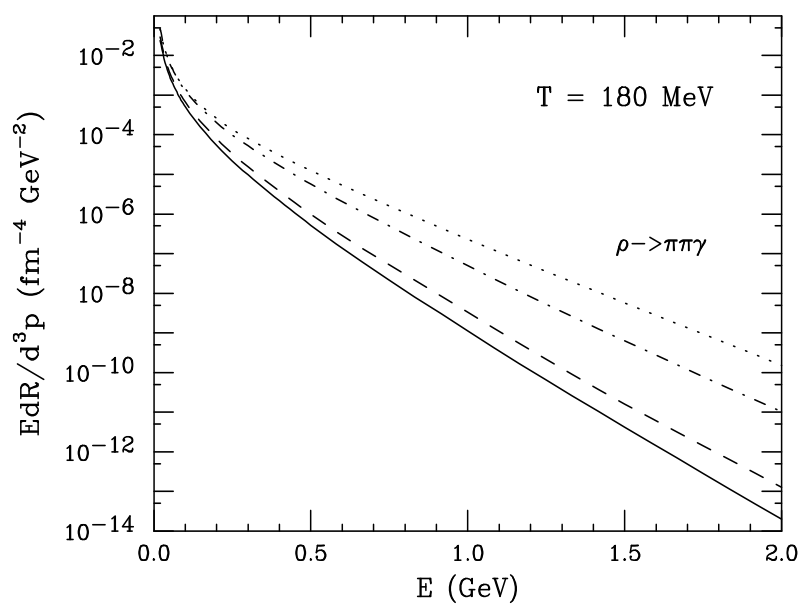

FIG. 13. Same as Fig. 12 at $T=180 \mathrm{MeV}$.

Since the lifetimes of the rho and omega mesons are comparable to the strong interaction time scale it is necessary to consider their decay channels. The possibility of detecting a high energy photon from the decay $\rho \rightarrow \pi \pi \gamma$ reduces as the mass of the rho decreases with increasing baryon density. This is clearly observed from Fig. (12) at $T=160$ $\mathrm{MeV}$. The corresponding results for $T=180 \mathrm{MeV}$ (Fig. (13)) shows a similar nature. In Figs. (14) and (15) we display the photon spectra from omega decay for $T=160$ and $180 \mathrm{MeV}$ respectively. The medium effects in this case are rather small. 


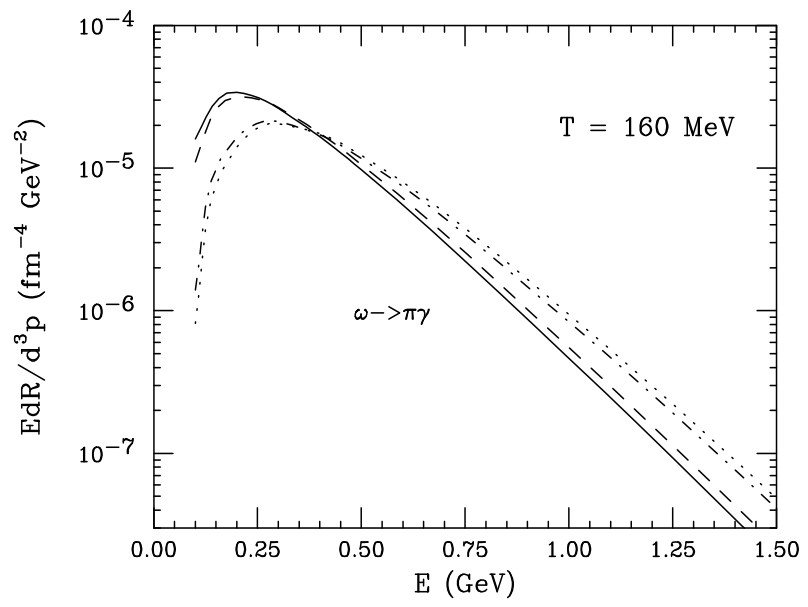

FIG. 14. Same as Fig. 8 for the decay $\omega \rightarrow \pi \gamma$.

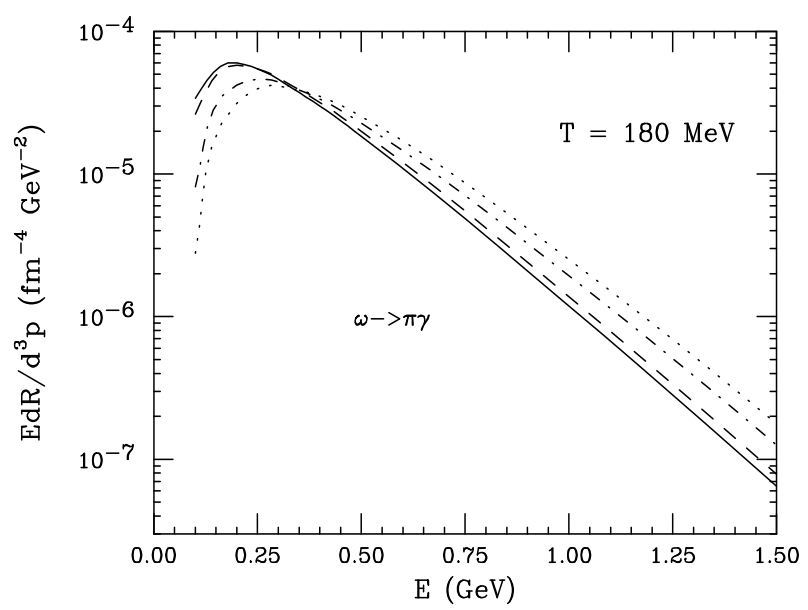

FIG. 15. Same as Fig. 14 at $T=180 \mathrm{MeV}$.

Finally, the total photon emission rate at $T=160 \mathrm{MeV}$ is plotted in Fig. (16) as a function of the energy of the emitted photon for various values of baryon density. At twice normal nuclear matter density the photon yield is seen to be higher by a factor of $\sim 4$ compared to the case when the effects of the thermal bath on the hadronic properties are neglected. This is true for almost the entire energy range of the emitted photon under consideration. A qualitatively similar trend is observed at $T=180 \mathrm{MeV}$ as shown in Fig. (17). As mentioned earlier, the delta-hole polarization effects on pion and its consequences on photon spectra may be important which has been neglected in the present work.

In order to accout for the fact that most of the photon producing reactions which we have considered contain unstable particles (rho and omega) in the external lines we have folded the emission rate with the appropriate spectral function of the unstable particle as given in eqs. (11) and (12). The limits of the $s$ integration in the case of different channel have been determined from kinematical considerations. The results for the reactions $\pi \pi \rightarrow \rho \gamma$, $\pi \pi \rightarrow \pi \gamma, \rho \rightarrow \pi \pi \gamma$ and $\omega \rightarrow \pi \gamma$ have been shown in Fig. (18). The difference caused by the inclusion of the spectral function is observed to be $\sim$ a few percent. This is reflected in the total photon spectra as shown in Fig. (19).

From the set of photon producing reactions considered in this work it is clear that the rho and not the omega meson plays the dominant role. Therefore the separation of the longitudinal and transverse mode in case of omega meson has negligible effect on the total photon emission rate. In our calculation the full energy momentum dependence of the self 
energy of rho enters through the propagator (eq. (7)). However, we have observed that the quantity $k_{0}^{2}-\mathbf{k}^{2}$ along the dispersion curve (Fig. (3)) remains almost constant $\left(\sim m_{\rho}^{* 2}\right.$, which is defined as $k_{0}^{2}$ at $\mathbf{k}^{2}=0$ of the mass hyperbola). In other words in this particular case as far as the photon emission rate is concerned a simple pole approximation of the rho propagator at $k^{2}=m_{\rho}^{2}$ reproduces the results obtained with the full propagator.

So far we have studied the effects of in-medium hadronic masses and decay widths on the photon spectra, where, the latter is found to have negligible effects. However, the modification in the rho decay width due to BE, arising from the induced emission of pions in the thermal medium, plays a crucial role in low mass lepton pair production. It has been shown in Ref. 32] that this effect arises naturally in a calculation based on finite temperature field theory.

For the sake of illustration, we consider the effect of thermal nucleon loop on the lepton pair production from pion annihilation via (rho mediated) vector meson dominance. In the work of Li, Ko and Brown [22], the observed enhancement on the low mass dilepton yield was attributed solely to the the matter induced mass modification of the meson, while neglecting the BE effect. In Fig. (20) the dilepton emission rate is plotted as a function of invariant mass at a temperature $T=180 \mathrm{MeV}$. It is clear from the figure that the inclusion of BE effects reduces the yield by increasing the decay width. Quantitatively, a suppression by a factor of 3 is observed at the invariant mass, $M=m_{\rho}^{*}$.

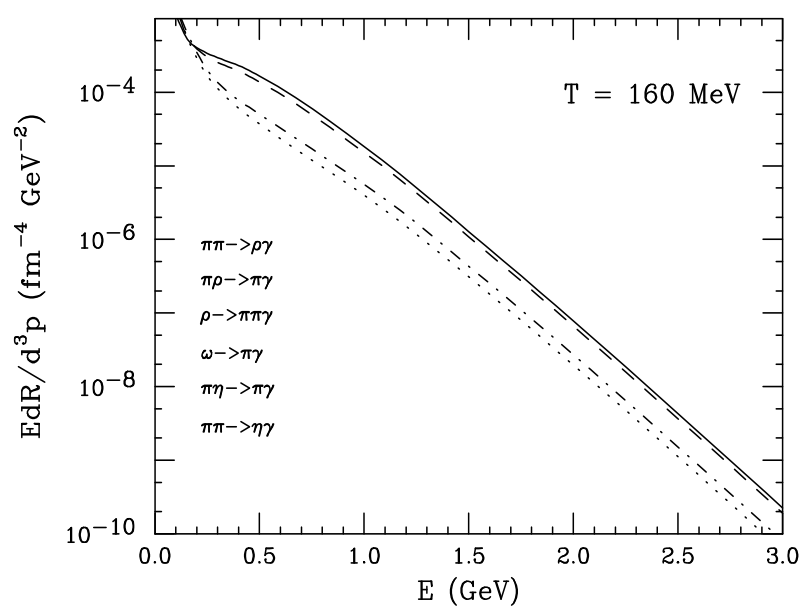

FIG. 16. Total photon emission rates as a function of energy of the emitted photon at $T=160 \mathrm{MeV}$. Solid and dashed lines show the results for $\rho / \rho_{0}=2$ and 1 respectively. The dotdashed line represents only finite temperature effects and dotted line shows the result when no medium effect is considered.

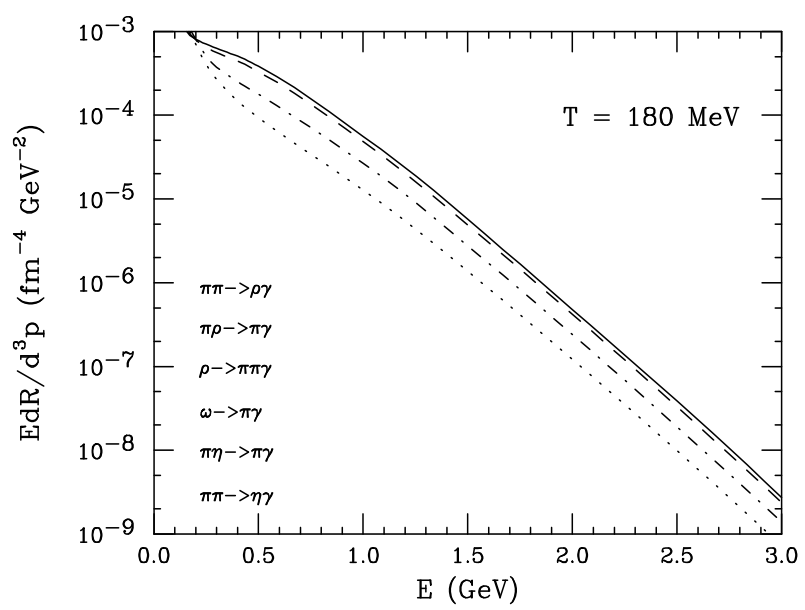

FIG. 17. Same as Fig. 16 at $T=180 \mathrm{MeV}$. 


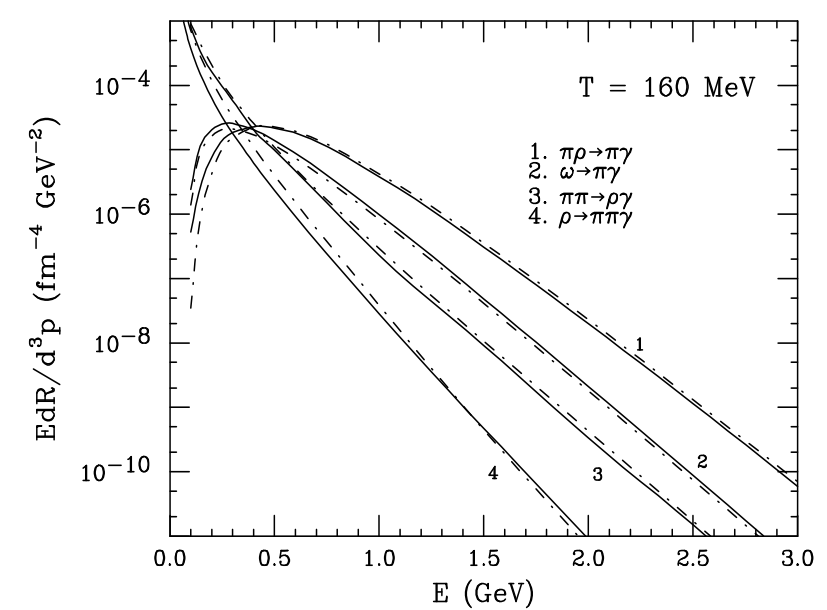

FIG. 18. Effect of spectral function of vector mesons on photon emission rates at $T=160 \mathrm{MeV}$ and zero baryon density. Solid (dotdashed) line shows results with (without) the inclusion of spectral function.

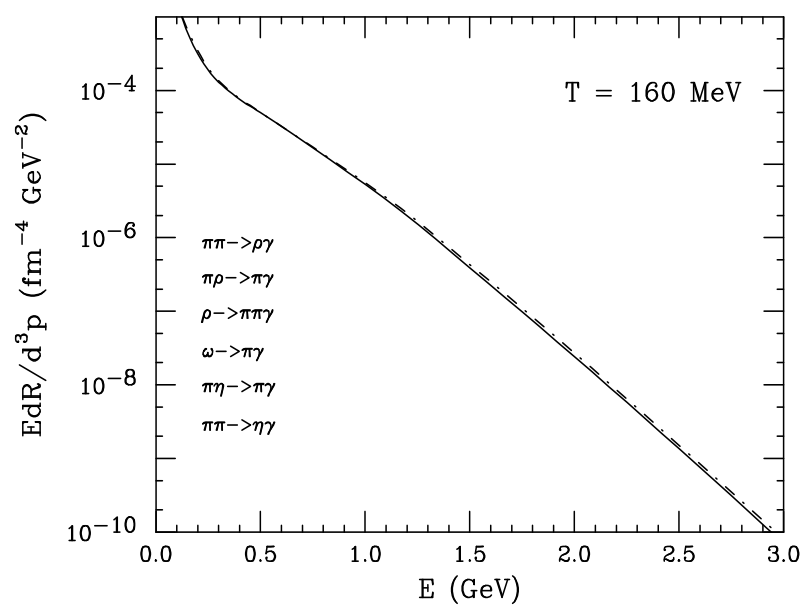

FIG. 19. Total photon emission rate at $T=160 \mathrm{MeV}$ and zero baryon density.

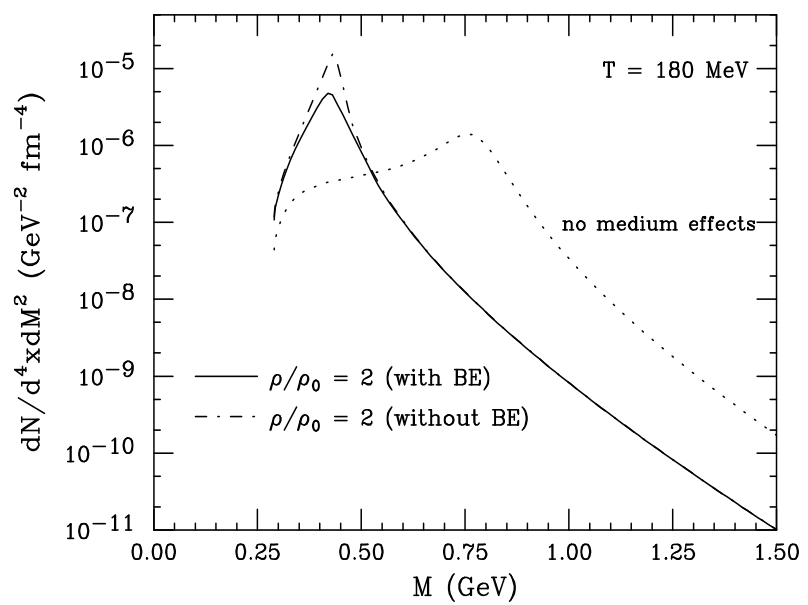

FIG. 20. Dilepton emission rates as a function of invariant mass at $T=180 \mathrm{MeV}$ and twice nuclear matter density. Solid and dotdashed lines show the results with and without BE effects respectively. The dotted line shows the result when no medium effect is considered. 


\section{SUMMARY AND DISCUSSIONS}

In this work we have studied the variation of effective masses and decay widths of nucleons and vector mesons at non-zero temperature and baryon density within the framework of an effective Lagrangian approach. It is seen that the nucleon mass for non-zero baryon density increases slightly with temperature before dropping. We have seen that the effect of the vacuum fluctuation corrections to MFT value of nucleon mass is substantial at higher values of the baryon density. However, the consequence of such an effect on the vector meson mass is minimal.

We have also estimated the photon emission rate from a hot and dense hadronic matter. The inclusion of in-medium properties of hadrons enhances the emission rate. Although the effect of the decay width of the rho meson on photon spectra is small, it affects the dilepton emission rate quite substantially in the low invariant mass region (see e.g. Ref. [33). An exhaustive work taking into account all the important dilepton producing channels will be published shortly.

So far we have talked about the static (fixed temperature) emission rate of photons and dileptons. The calculated spectra has to be folded with the space time evolution dynamics. This requires a self consistent calculation of the equation of state, which is a fundamental ingredient to any hydrodynamical calculation. Because of the substantial change in hadronic properties at finite temperature and density a simple Bjorken-like [34 hydrodynamical model appears to be grossly inadequate. A rigorous calculation addressing these issues is in progress.

\section{Acknowledgement:}

We are grateful to Professor Binayak Dutta-Roy for many useful discussions and also to the referee(s) whose comments were very helpful.

\section{APPENDIX A:}

The polarisation tensor of a vector (omega or rho) meson due to $V N N$ interaction is obtained from the Lagrangian described by eq. (9) as

$$
\Pi^{\mu \nu}=-2 i g_{V N N}^{2} \int \frac{d^{4} p}{(2 \pi)^{4}} \operatorname{Tr}\left[\Gamma^{\mu}(k) G(p) \Gamma^{\nu}(-k) G(p+k)\right]
$$

where

$$
\Gamma^{\mu}(k)=\gamma^{\mu}+i \frac{\kappa_{V}}{2 M} \sigma^{\mu \alpha} k_{\alpha}
$$

In a hot and dense medium moving with four velocity $u^{\mu}$ the fermion propagator is given by 35

$$
\begin{aligned}
G(p) & =\left(\gamma^{\mu} p^{\prime}{ }_{\mu}+M^{*}\right)\left[\frac{1}{p^{2}-M^{* 2}+i \epsilon}+2 \pi i \frac{\delta\left(p^{2}-M^{* 2}\right)}{e^{|p \cdot u|}+1}\right] \\
& =G_{F}(p)+G_{D}(p)
\end{aligned}
$$

with $p^{\nu}=p^{\nu}+\mu^{*} u^{\nu}$ and $|p \cdot u|=\sqrt{|\mathbf{p}|^{2}+\mathbf{M}^{* 2}}-\mu^{*}$.

The vacuum part of the vector meson self energy due to $N \bar{N}$ polarization in the modified Dirac sea is given by

$$
\Pi_{\mu \nu}^{F}=-2 i g_{V N N}^{2} \int \frac{d^{4} p}{(2 \pi)^{4}} \operatorname{Tr}\left[\Gamma^{\mu}(k) G_{F}(p) \Gamma^{\nu}(-k) G_{F}(p+k)\right]
$$

with 


$$
\begin{aligned}
\Pi^{F}\left(k^{2}\right) & =\frac{1}{3} \operatorname{Re}\left(\Pi^{F}\right)_{\mu}^{\mu} \\
& =-\frac{g_{V N N}^{2}}{\pi^{2}} k^{2}\left[I_{1}+M^{*} \frac{\kappa_{V}}{2 M} I_{2}+\frac{1}{2}\left(\frac{\kappa_{V}}{2 M}\right)^{2}\left(k^{2} I_{1}+M^{* 2} I_{2}\right)\right]
\end{aligned}
$$

where,

$$
\begin{gathered}
I_{1}=\int_{0}^{1} d z z(1-z) \ln \left[\frac{M^{* 2}-k^{2} z(1-z)}{M^{2}-k^{2} z(1-z)}\right], \\
I_{2}=\int_{0}^{1} d z \ln \left[\frac{M^{* 2}-k^{2} z(1-z)}{M^{2}-k^{2} z(1-z)}\right] .
\end{gathered}
$$

Here, it is important to note that we have used the in-medium mass obtained by solving Eqs. (5) and (6) simultaneously. The renormalization scheme employed by Hatsuda et al. [36]. has been adopted here.

In a hot and dense medium, because of Lorentz invariance and current conservation the general structure of the polarisation tensor is of the form

$$
\Pi^{\mu \nu}=\Pi_{T}\left(k_{0},|\mathbf{k}|\right) \mathbf{A}^{\mu \nu}+\Pi_{\mathbf{L}}\left(\mathbf{k}_{\mathbf{0}},|\mathbf{k}|\right) \mathbf{B}^{\mu \nu}
$$

where the two Lorentz invariant functions $\Pi_{T}$ and $\Pi_{L}$ are obtained by contraction:

$$
\begin{aligned}
& \Pi_{L}=-\frac{k^{2}}{|\mathbf{k}|^{2}} u^{\mu} u^{\nu} \Pi_{\mu \nu} \\
& \Pi_{T}=\frac{1}{2}\left(\Pi_{\mu}^{\mu}-\Pi_{L}\right)
\end{aligned}
$$

In the case of the vector meson interacting with real particle-hole excitations in the nuclear medium these are given by

$$
\begin{aligned}
\Pi_{\mu \nu}^{D} & =-2 i g_{V N N}^{2} \int \frac{d^{4} p}{(2 \pi)^{4}} \operatorname{Tr}\left[\Gamma^{\mu}(k) G_{F}(p) \Gamma^{\nu}(-k) G_{D}(p+k)+(F \leftrightarrow D)\right] \\
& =\left(\Pi_{v}^{D}+\Pi_{v t}^{D}+\Pi_{t}^{D}\right)_{\mu \nu}
\end{aligned}
$$

with

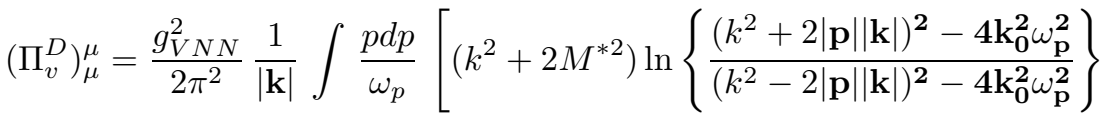

$$
\begin{aligned}
& -8|\mathbf{p}||\mathbf{k}|]\left[n_{B}\left(\mu^{*}, T\right)+\bar{n}_{B}\left(\mu^{*}, T\right)\right] \\
& \left(\Pi_{v t}^{D}\right)_{\mu}^{\mu}=\frac{3 g_{V N N}^{2}}{\pi^{2}} M^{*}\left(\frac{\kappa_{V}}{2 M}\right) \frac{k^{2}}{|\mathbf{k}|} \int \frac{p d p}{\omega_{p}} \ln \left\{\frac{\left(k^{2}+2|\mathbf{p}||\mathbf{k}|\right)^{2}-4 \mathbf{k}_{\mathbf{0}}^{\mathbf{2}} \omega_{\mathbf{p}}^{\mathbf{2}}}{\left(k^{2}-2|\mathbf{p}||\mathbf{k}|\right)^{\mathbf{2}}-\mathbf{4} \mathbf{k}_{\mathbf{0}}^{\mathbf{2}} \omega_{\mathbf{p}}^{\mathbf{2}}}\right\} \\
& \times\left[n_{B}\left(\mu^{*}, T\right)+\bar{n}_{B}\left(\mu^{*}, T\right)\right] \\
& \left(\Pi_{t}^{D}\right)_{\mu}^{\mu}=\frac{g_{V N N}^{2}}{4 \pi^{2}}\left(\frac{\kappa_{V}}{2 M}\right)^{2} \frac{k^{2}}{|\mathbf{k}|} \int \frac{p d p}{\omega_{p}}\left[\left(k^{2}+8 M^{* 2}\right)\right.
\end{aligned}
$$

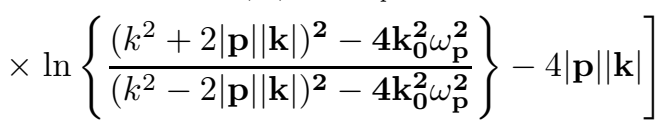

$$
\begin{aligned}
& \times\left[n_{B}\left(\mu^{*}, T\right)+\bar{n}_{B}\left(\mu^{*}, T\right)\right]
\end{aligned}
$$


The longitudinal component of the polarisation tensor is given by

$$
\Pi_{L}^{D}=\Pi_{L}^{D, v}+\Pi_{L}^{D, v t}+\Pi_{L}^{D, t}
$$

with

$$
\begin{aligned}
& \Pi_{L}^{D, v}=-\frac{g_{V N N}^{2}}{4 \pi^{2}} \frac{k^{2}}{|\mathbf{k}|^{3}} \int \frac{p d p}{\omega_{p}}\left[\left\{\left(k_{0}-2 \omega_{p}\right)^{2}-|\mathbf{k}|^{2}\right\} \ln \frac{\mathbf{k}^{\mathbf{2}}-\mathbf{2} \mathbf{k}_{\mathbf{0}} \omega_{\mathbf{p}}+\mathbf{2}|\mathbf{p}||\mathbf{k}|}{\mathbf{k}^{\mathbf{2}}-\mathbf{2} \mathbf{k}_{\mathbf{0}} \omega_{\mathbf{p}}-\mathbf{2}|\mathbf{p}||\mathbf{k}|}\right. \\
& \left.+\left\{\left(k_{0}+2 \omega_{p}\right)^{2}-|\mathbf{k}|^{2}\right\} \ln \frac{\mathbf{k}^{2}+\mathbf{2} \mathbf{k}_{\mathbf{0}} \omega_{\mathbf{p}}+\mathbf{2}|\mathbf{p}||\mathbf{k}|}{\mathbf{k}^{\mathbf{2}}+\mathbf{2} \mathbf{k}_{\mathbf{0}} \omega_{\mathbf{p}}-\mathbf{2}|\mathbf{p}||\mathbf{k}|}-\mathbf{8}|\mathbf{p}||\mathbf{k}|\right] \\
& \times\left[n_{B}\left(\mu^{*}, T\right)+\bar{n}_{B}\left(\mu^{*}, T\right)\right] \\
& \Pi_{L}^{D, v t}=\frac{g_{V N N}^{2}}{\pi^{2}} M^{*}\left(\frac{\kappa_{V}}{2 M}\right) \frac{k^{2}}{|\mathbf{k}|} \int \frac{p d p}{\omega_{p}} \ln \left\{\frac{\left(k^{2}+2|\mathbf{p}||\mathbf{k}|\right)^{2}-4 \mathbf{k}_{\mathbf{0}}^{\mathbf{2}} \omega_{\mathbf{p}}^{\mathbf{2}}}{\left(k^{2}-2|\mathbf{p}||\mathbf{k}|\right)^{\mathbf{2}}-\mathbf{4} \mathbf{k}_{\mathbf{0}}^{\mathbf{2}} \omega_{\mathbf{p}}^{\mathbf{2}}}\right\} \\
& \times\left[n_{B}\left(\mu^{*}, T\right)+\bar{n}_{B}\left(\mu^{*}, T\right)\right] \\
& \Pi_{L}^{D, t}=-\frac{g_{V N N}^{2}}{2 \pi^{2}}\left(\frac{\kappa_{V}}{2 M}\right)^{2} \frac{k^{2}}{|\mathbf{k}|} \int \frac{p d p}{\omega_{p}}\left[\left\{2|\mathbf{p}|^{\mathbf{2}}-\frac{\mathbf{k}^{\mathbf{2}}}{\mathbf{2}}-\frac{\left(\mathbf{k}^{\mathbf{2}}-\mathbf{2} \mathbf{k}_{\mathbf{0}} \omega_{\mathbf{p}}\right)^{\mathbf{2}}}{\mathbf{2}|\mathbf{k}|^{\mathbf{2}}}\right\}\right. \\
& \times \ln \frac{k^{2}-2 k_{0} \omega_{p}+2|\mathbf{p}||\mathbf{k}|}{k^{2}-2 k_{0} \omega_{p}-2|\mathbf{p}||\mathbf{k}|}+\left\{2|\mathbf{p}|^{2}-\mathbf{k}^{2}-\frac{\left(\mathbf{k}^{2}+\mathbf{2} \mathbf{k}_{\mathbf{0}} \omega_{\mathbf{p}}\right)^{2}}{|\mathbf{k}|^{\mathbf{2}}}\right\} \\
& \left.\times \ln \frac{k^{2}+2 k_{0} \omega_{p}+2|\mathbf{p}||\mathbf{k}|}{k^{2}+2 k_{0} \omega_{p}-2|\mathbf{p}||\mathbf{k}|}-\frac{4|\mathbf{p}| \mathbf{k}_{\mathbf{0}}^{2}}{|\mathbf{k}|}\right] \\
& \times\left[n_{B}\left(\mu^{*}, T\right)+\bar{n}_{B}\left(\mu^{*}, T\right)\right]
\end{aligned}
$$

The dispersion relation for the longitudinal (transverse) mode now reads

$$
k_{0}^{2}-|\mathbf{k}|^{2}-\mathbf{m}_{\mathbf{V}}^{2}+\operatorname{Re} \boldsymbol{\Pi}_{\mathbf{L}(\mathbf{T})}^{\mathrm{D}}\left(\mathbf{k}_{\mathbf{0}}, \mathbf{k}\right)+\operatorname{Re} \boldsymbol{\Pi}^{\mathbf{F}}\left(\mathbf{k}^{\mathbf{2}}\right)=\mathbf{0}
$$

Usually the physical mass $\left(m_{V}^{*}\right)$ is defined as the lowest zero of the above equation in the limit $\mathbf{k} \rightarrow 0$. In this limit $\Pi_{T}^{D}=\Pi_{L}^{D}=\Pi^{D}$, and we have,

$$
\frac{1}{3} \Pi_{\mu}^{\mu}=\Pi=\Pi^{D}+\Pi^{F}
$$

where

$$
\Pi^{D}(\omega, \mathbf{k} \rightarrow 0)=-\frac{4 g_{V N N}^{2}}{\pi^{2}} \int p^{2} d p F\left(|\mathbf{p}|, \mathbf{M}^{*}\right)\left[\mathbf{n}_{\mathbf{B}}\left(\mu^{*}, \mathbf{T}\right)+\overline{\mathbf{n}}_{\mathbf{B}}\left(\mu^{*}, \mathbf{T}\right)\right]
$$

with

$$
\begin{aligned}
F\left(|\mathbf{p}|, \mathbf{M}^{*}\right)= & \frac{1}{\omega_{p}\left(4 \omega_{p}^{2}-\omega^{2}\right)}\left[\frac{2}{3}\left(2|\mathbf{p}|^{\mathbf{2}}+\mathbf{3} \mathbf{M}^{* \mathbf{2}}\right)+\omega^{\mathbf{2}}\left\{\mathbf{2} \mathbf{M}^{*}\left(\frac{\kappa_{\mathbf{V}}}{\mathbf{2} \mathbf{M}}\right)\right.\right. \\
& \left.\left.+\frac{2}{3}\left(\frac{\kappa_{V}}{2 M}\right)^{2}\left(|\mathbf{p}|^{\mathbf{2}}+\mathbf{3} \mathbf{M}^{* \mathbf{2}}\right)\right\}\right]
\end{aligned}
$$

where $\omega_{p}^{2}=\mathbf{p}^{2}+M^{* 2}$.

The effective mass of the vector meson is then obtained by solving the equation:

$$
k_{0}^{2}-m_{V}^{2}+\operatorname{Re} \Pi=0
$$


One finds reference to two other kinds of masses in the literature. The invariant mass is defined as the lowest order zero of eq. (A18) with $\Pi^{D}$ neglected. Again, the screening mass of a vector meson is obtained from the pure imaginary zero of the quantity on the left hand side of the same equation with $k_{0}=0$.

The imaginary part of the self energy of a particle is related to the probability of its survival in a medium at finite temperature. For a rho meson propagating with energy $\omega>0$ and three momentum $\mathbf{k}$ this is given by

$$
\Gamma(\omega)=\frac{g_{\rho \pi \pi}^{2}}{48 \pi} W^{3}(s) \frac{s}{\omega}\left[1+\frac{2 T}{W(s) \sqrt{\omega^{2}-s}} \ln \left\{\frac{1-\exp \left[-\frac{\beta}{2}\left(\omega+W(s) \sqrt{\omega^{2}-s}\right)\right]}{1-\exp \left[-\frac{\beta}{2}\left(\omega-W(s) \sqrt{\omega^{2}-s}\right)\right]}\right\}\right]
$$

where $s=k^{2}=\omega^{2}-\mathbf{k}^{2}$ and $W(s)=\sqrt{1-4 m_{\pi}^{2} / s}$. In the limit $|\mathbf{k}| \rightarrow \mathbf{0}$, the above expression reduces to the in-medium decay width and is given by

$$
\Gamma_{\rho \rightarrow \pi \pi}=\frac{g_{\rho \pi \pi}^{2}}{48 \pi} \omega W^{3}(\omega)\left[\left(1+n\left(\frac{\omega}{2}\right)\right)\left(1+n\left(\frac{\omega}{2}\right)\right)-n\left(\frac{\omega}{2}\right) n\left(\frac{\omega}{2}\right)\right]
$$

where $\omega=m_{\rho}^{*}$ is the in-medium mass of the rho due to $N-N$ interaction.

\section{APPENDIX B:}

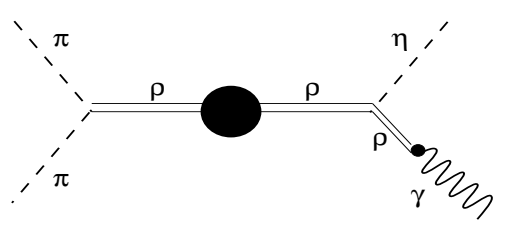

(a)

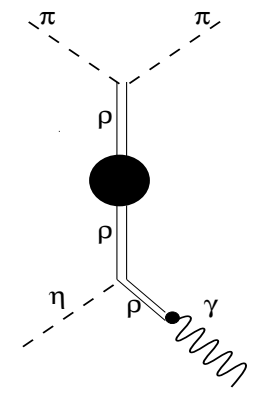

(b)

FIG. 21. Feynman diagrams for $\pi \pi \rightarrow \eta \gamma$ and $\pi \eta \rightarrow \pi \gamma$.

The $\rho \pi \eta$ interaction is described by Eq. (15). The invariant amplitude for the reaction $\pi^{+}\left(p_{1}\right)+\pi^{-}\left(p_{2}\right) \rightarrow$ $\eta\left(p_{3}\right)+\gamma\left(p_{4}\right)$ is given by

$$
\left|\mathcal{M}_{a}\right|^{2}=\frac{4 \pi \alpha g_{\rho \rho \eta}^{2}}{m_{\eta}^{2}\left[\left(s-m_{\rho}^{2}\right)^{2}+m_{\rho}^{2} \Gamma_{\rho}^{2}\right]}\left[s\left(u-m_{\pi}^{2}\right)\left(t-m_{\pi}^{2}\right)-m_{\pi}^{2}\left(s-m_{\eta}^{2}\right)^{2}\right]
$$

The Feynman diagram for the above process is shown in Fig. (21a). For the reaction $\pi^{ \pm}\left(p_{1}\right)+\eta\left(p_{2}\right) \rightarrow \pi^{ \pm}\left(p_{3}\right)+\gamma\left(p_{4}\right)$ (see Fig. (21b)),

$$
\left|\mathcal{M}_{b}\right|^{2}=\frac{4 \pi \alpha g_{\rho \rho \eta}^{2}}{m_{\eta}^{2}\left[\left(t-m_{\rho}^{2}\right)^{2}+m_{\rho}^{2} \Gamma_{\rho}^{2}\right]}\left[t\left(u-m_{\pi}^{2}\right)\left(s-m_{\pi}^{2}\right)-m_{\pi}^{2}\left(t-m_{\eta}^{2}\right)^{2}\right]
$$

The coupling constant $g_{\rho \rho \eta}$ is evaluated from the following relations:

$$
\Gamma(\rho \rightarrow \eta \gamma)=\frac{\left(m_{\rho}^{2}-m_{\eta}^{2}\right)^{3}}{96 \pi m_{\eta}^{2} m_{\rho}^{3}} g_{\eta \gamma \rho}^{2}
$$




$$
\begin{aligned}
g_{\eta \gamma \rho} & =\frac{e g_{\rho \rho \eta}}{g_{\rho \pi \pi}} \\
\frac{g_{\rho \pi \pi}^{2}}{4 \pi} & =2.9
\end{aligned}
$$

and $\Gamma(\rho \rightarrow \eta \gamma)=(57 \pm 10.5) \mathrm{keV}$

[1] A. Ukawa, in Quark Matter'97, Tsukuba, Japan (Proc. to be published in Nucl. Phys. A).

[2] R. C. Hwa (ed.), Quark-Gluon Plasma, World Scientific, Singapore 1990.

[3] J. Alam, S. Raha, and B. Sinha, Phys. Rep. 273243 (1996).

[4] R. D. Pisarski, hep-ph/9503330.

[5] U. Meissner, Phys. Rep. 161213 (1988).

[6] R. J. Furnstahl and T. Hatsuda, Phys. Rev. D42 1744 (1990); C. Adami, T. Hatsuda and I. Zahed, Phys. Rev. D43 921 (1991); T. Hatsuda, Nucl. Phys. A544 27c (1992).

[7] G. E. Brown, Nucl. Phys. A522 397c (1991).

[8] G. E. Brown and M. Rho, Phys. Rev. Lett. 662720 (1991).

[9] A. Bhattacharyya, J. Alam, S. Raha and B. Sinha, Int. J. Mod. Phys. A12 5639 (1997).

[10] B. D. Serot and J. D. Walecka, Advances in Nuclear Physics Vol. 16 Plenum Press, New York 1986.

[11] S. A. Chin, Ann. Phys. 108301 (1977).

[12] S. Sarkar, J. Alam, P. Roy, A. K. Dutt-Majumder, B. Dutta-Roy and B. Sinha, Nucl. Phys. A634 206 (1998).

[13] See for example, Nucl. Phys. A610 (1996).

[14] H. A. Weldon, Ann. Phys. bf 22843 (1993).

[15] J. Alam, S. Sarkar, P. Roy, B. Dutta-Roy and B. Sinha Phys. Rev. C59 905 (1999).

[16] M. Le Bellac, Thermal Field Theory, (Cambridge University Press, N. Y., 1996)

[17] A. A. Abrikosov, L. P. Gor'kov and I. E. Dzyaloshinski, Method of Quantum Field Theory in Statistical Physics, (Prentice Hall, Engelwood Cliffs, N. J. 1963).

[18] D. N. Zubarev, Sov. Phys. Uspekhi, 369 (1960).

[19] R. Rapp, G. Chanfray and J. Wambach, Nucl. Phys. A617 472 (1997).

[20] J. J. Sakurai, Currents and Mesons, The University of Chicago Press, Chicago, 1969.

[21] H. C. Jean, J. Piekarewicz and A. G. Williams, Phys. Rev. C49, 1981(1994).

[22] G. Q. Li, C. M. Ko and G. E. Brown, Nucl. Phys. A606 568 (1996).

[23] J. I. Kapusta, Finite Temperature Field Theory, Cambridge University Press, 1993.

[24] T. Hatsuda, private communication.

[25] C. Gale and J. I. Kapusta, Nucl. Phys. B357 65 (1991).

[26] F. Klingl, N. Kaiser and W. Weise, Nucl. Phys. A624 527 (1996).

[27] M. Asakawa, C. M. Ko, P. Levai and X. J. Qiu Phys. Rev. C46 R1159 (1992).

[28] M. Herrmann, B. L. Friman and W. Nörenberg, Nucl. Phys. A560 411 (1993).

[29] G. Chanfray and P. Shuck, Nucl. Phys. A545 271c (1992).

[30] A. K. Dutt-Mazumder, J. Alam, B. Dutta-Roy and Bikash Sinha, Phys. Lett. B378 35 (1996).

[31] J. Kapusta, P. Lichard, and D. Seibert, Phys. Rev. D44 2774 (1991).

[32] H. A. Weldon, Phys. Rev. D28 2007 (1983).

[33] C. Song and C. M. Ko, Phys. Rev. C53 2371 (1996).

[34] J. D. Bjorken, Phys. Rev. D27 140 (1983).

[35] H. A. Weldon, Phys. Rev. D26 1394 (1982).

[36] T. Hatsuda, H. Shiomi and H. Kuwabara, Prog. Th. Phys. 951009 (1996). 